\title{
Focal Tuberculin Reaction (FTR) in Bone and Joint Tuberculosis
}

\author{
By \\ Saburo Iino \\ (飯 野三 郎) \\ From the Orthopedic-Surgical Department, Tohoku University, Sendai; \\ Director: Prof. S. Iino \\ (Received for publication, July 4, 1955)
}

It is well known that a tuberculously sensitized individual comes to possess a state of specific tuberculous allergy generally affecting its whole organism which may be called humoral or generalized allergy, and this fact is utilized in clinical practice in distinguishing between tuberculously sensitized and non-sensitized individuals in the form of intracutaneous tuberculin reaction (the Mantoux's reaction). It has, however, not yet been sufficiently studied whether in a sensitized case with present or past tuberculous foci the allergic states in the vicinity of such foci might show some different reactions from those in other portions distant from the foci. If such a difference of reaction in different portions is to be demonstrated by tuberculosis allergy tests such as the intracutaneous tuberculin test, it should be most advantageous for study to select cases of a disease with its foci situated most near to the skin and not histologically disconnected from it, and moreover show a considerable activity, such as bone or joint tuberculosis. The author, based on such an opinion, and postulating that the reactions in the portions near to and distant from the foci should show some specific difference, has made experiments to compare the intracutaneous tuberculin reaction in the teguments of the foci and at portions distant from these since 1947,3$), 4,5)$ and has succeeded in demonstrating that there is, in fact, such a difference. Nearly all the experimental procedures in this study have been carried out by my co-worker Dr. M. Kimura.

\section{EXPERIMENTAL}

\section{Preliminary Experiments}

435 normal adult subjects, male and female, with their tuberculin reaction turned positive, were tested for the same reaction at 26 portions in different parts of the body. In these tests, $0.1 \mathrm{cc}$. each of old tuberculin diluted to 2,000 folds in volume was intracutaneously injected at the in- 
fraclavicular region, the mamillary region, the costal arch, the shoulder joint, the upper arm, the fore arm, the wrist joint, the intrascapular region, the sacroiliac region, the hip-joint, the thigh, the knee-joint, the lower leg, the ankle joint etc., always on both sides, right and left, symmetrically. The reaction 48 hours after the injection was as follows: the difference of the mean diameters of the reddened areas in the corresponding portions on both sides was below $3.0 \mathrm{~mm}$. in most of the cases and $2.5 \mathrm{~mm}$. in average, except in the upper and the fore-arms, where the mean difference amounted to $3.1 \mathrm{~mm}$., probably due to repeated tuberculin tests or BCG injections prior to the tests in question. From these preliminary tests, we could conclude that the intracutaneous tuberculin reaction in normal subjects rarely shows a difference exceeding $3.0 \mathrm{~mm}$. in the mean diameter of reddened area in symmetrically corresponding portions of the body, except in the arms.

\section{Principal Experiments}

Taking 178 cases of non-tuberculous diseases of bone and joint and 189 cases of bone and joint tuberculosis as subjects, $0.1 \mathrm{cc}$. of the old tuberculin in 2,000 fold dilution was injected into the skin 1) covering the foci, 2) on the exactly corresponding portion on the contralateral side (when the focus was in the vertebrae, in the skin over a healthy vertebra at a distance from it) and 3) on the volar side of the forearm. The mean diameter of the reddened area was measured 48 hours after the injection and the difference in diameter between the healthy and the affected side, expressed in $\mathrm{mm}$, was taken as the value of the focal tuberculin reactions (hereunder referred to as FTR). When the diameter was larger on the affected than on the healthy side, the value was signed $(+)$ and when vice versa $(-)$. Since the difference by sides is below $3.0 \mathrm{~mm}$. in normal cases as shown in the preliminary experiments, the FTR values between \pm 3.0 and 0 were considered indifferent and expressed as FTR (ind.) and values exceeding \pm 3.0 were taken as significant and expressed as FTR (dif. + ) or FTR (dif. -).

The results of this experiment were as follows (Table I). In the group of non-tuberculous cases, 145 or $81.5 \%$ of the total 178 had FTR (ind.), only 5.6\% FTR (dif. +) and $12.9 \%$ FTR (dif. -). If from these cases of FTR (dif.), 38 cases with rheumatic diseases with rheumatic foci, which in themselves are taken to be, in a sense, special states of tuberculous allergy, because of its essential influences upon the local allergy are excluded, the rate of FTR (ind.) among the non-tuberculous cases of bone and joint rises to $86.4 \%$ and the rate of FTR (dif. +) is reduced to $4.3 \%$ and that of FTR (dif. -) to $9.3 \%$. Among these rheumatic cases, the rates are found to be FTR (ind.): $63.1 \%$, FTR (dif. + ) : $10.5 \%$ and 
FTR (dif. -) : 26.3\%. This heightened rate of FTR (dif.) in the rheumatic cases seems to possess a specific significance.

The FTR for 189 cases of bone and joint tuberculosis showed a reduced rate of (ind.) of $45.5 \%$, while those of (dif. +) and (dif. -) rose to $37.0 \%$ and $17.5 \%$ respectively or $54.5 \%$ (dif.) in total, a percentage nearly triple of that of non-tuberculous cases (18.5\%) (Table II). Above all it must be noted that in many of the FTR (dif.) cases in these tuberculous patients, the difference itself fluctuates rather widely: the cases with FTR $(+$ ) values between $5.1 \mathrm{~mm}$. and $7.0 \mathrm{~mm}$. (FTR + in our terminology) occupying $9.6 \%$, with the values between $7.1 \mathrm{~mm}$. and $9.0 \mathrm{~mm}$. (H) $7.4 \%$ and those with the values over $9.1 \mathrm{~mm}$. (H) ranging at $7.4 \%$, a finding much dissimilar to that obtained with non-tuberculous cases.

The above results suggest that our FTR could be considered to be a quantitative indicator of the specificity of tuberculous foci in joints and bones. The fact that the luetic osteoarthritis, a specific chronic inflammation with a certain affinity to tuberculosis, and chronic suppurative

TABLE I

FTR in Non-tuberculous Diseases

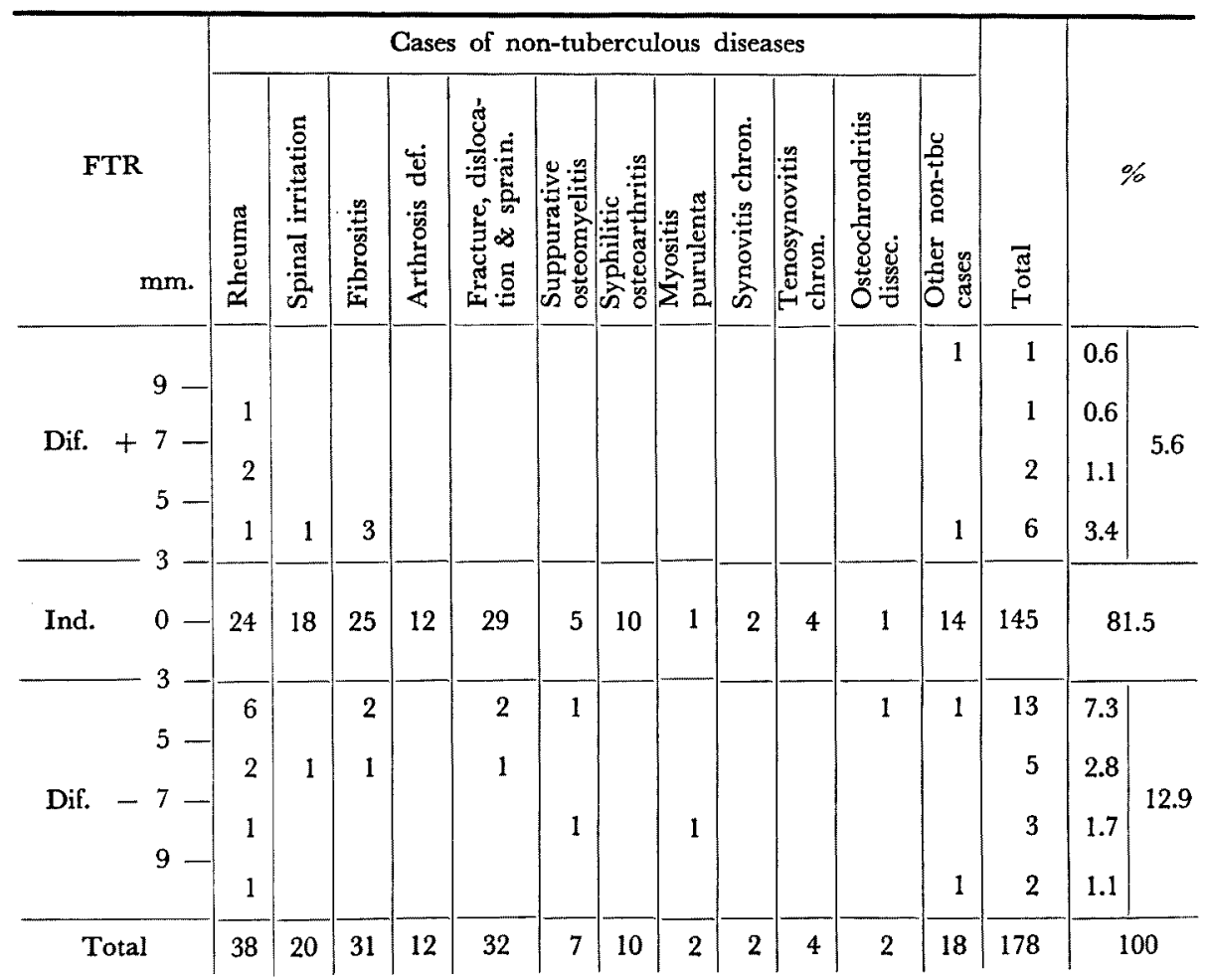


TABLE II

FTR in Bone and Joint Tuberculosis

\begin{tabular}{|c|c|c|c|c|c|c|c|c|c|c|c|c|c|}
\hline \multirow[b]{2}{*}{$\begin{array}{l}\text { FTR } \\
\text { mm. }\end{array}$} & \multicolumn{10}{|c|}{ Tuberculosis of the } & & \multirow{2}{*}{\multicolumn{2}{|c|}{$\%$}} \\
\hline & 总 & $\frac{0}{2}$ & 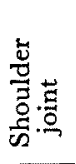 & 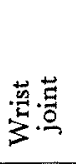 & 总 & 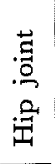 & 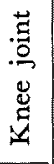 & $\frac{.}{\frac{.}{5}}$ & 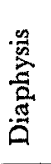 & 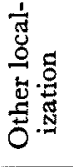 & $\stackrel{\pi}{0}$ & & \\
\hline $\begin{array}{r}9- \\
\text { Dif. }+7- \\
5-\end{array}$ & $\begin{array}{l}6 \\
9 \\
9 \\
8\end{array}$ & 2 & & 2 & 1 & $\begin{array}{l}2 \\
5\end{array}$ & $\begin{array}{l}3 \\
3 \\
7\end{array}$ & $\begin{array}{l}3 \\
1 \\
1\end{array}$ & 1 & 1 & $\begin{array}{l}14 \\
14 \\
18 \\
24\end{array}$ & $\begin{array}{r}7.4 \\
7.4 \\
9.6 \\
12.7\end{array}$ & 37.1 \\
\hline Ind. $\quad 0-$ & 46 & 5 & 4 & 1 & 6 & 5 & 8 & 3 & 5 & 3 & 86 & 45.5 & \\
\hline $\begin{array}{r}5- \\
\text { Dif. }-7- \\
9-\end{array}$ & $\begin{array}{l}8 \\
7 \\
3 \\
2\end{array}$ & 1 & & & $\begin{array}{l}1 \\
1\end{array}$ & $\begin{array}{l}2 \\
4\end{array}$ & 1 & & & 2 & $\begin{array}{r}13 \\
12 \\
6 \\
2\end{array}$ & $\begin{array}{l}6.9 \\
6.3 \\
3.2 \\
1.1\end{array}$ & 17.5 \\
\hline Total & 98 & 12 & 4 & 3 & 9 & 21 & 22 & 8 & 6 & 6 & 189 & 100 & \\
\hline
\end{tabular}

osteomyelitis cases show FTR (ind.) in nearly all cases, as shown in Table I, seems to emphasize this specificity for tuberculosis of this reaction.

Next, upon examining the change of FTR values according to the stage of the disease in 21 cases of tuberculous osteoarthritis whose current stage were apparent, it was found that among those in the initial stadium, $50 \%$ had FTR (ind.), 33.3\% (dif. +) and $16.7 \%$ (dif. - ), while in the florid stadium the rate of FTR (ind.) fell to $26.8 \%$ and of (dif. -) to $0.0 \%$ while that of FTR (dif. + ) rose to $71.5 \%$, and in the rest stadium, FTR (ind.) rerose to $66.7 \%$ while FTR (dif. + ) fell to $33.3 \%$ and FTR (dif. -) remained negative. Here, we see that FTR (dif. -) appears only in the initial stadium, FTR (dif. +) cases increase sharply in the florid stadium and redecrease in the rest stadium while FTR (ind.) increases in rate, a few seemingly indicating a parallelism between the local tuberculin allergy and the clinical progress of the disease. The nature and the conditions of the granulation tissue or fistula formation at the local focus also show an approximate parallelism with the FTR values. We have also investigated the serial changes of the FTR values by repeating the tests in all of 11 tuberculous osteoarthritic patients, and found that the FTR values begin to rise from the negative side in the intial stage to attain 
the maximum values in the florid stage and gradually turn to FTR (ind.) in the rest stadium, the right and left difference finally disappearing.

There was nearly no correlation perceptible between the blood sedimentation rate and FTR, endorsing the assumption of different factors responsible for these two phenomena respectively.

\section{Supplementary Experiments}

For the purpose to examine the possible influence upon the difference in FTR as stated above of non-specific responses of the tissue accompanying the process of focus formation, not representing specific, and local antigen-antibody reaction, such as exsudative process due to the abnormal capillary permeability, or changes of local diffusion or resorptive function, the following tests were made.

1) Capillar fragility test. Simultaneously with the FTR tests, tests for the capillary fragility (G-F) according to the method of Borbély were applied at neighboring portions. It resulted, as shown in Fig. 1, that

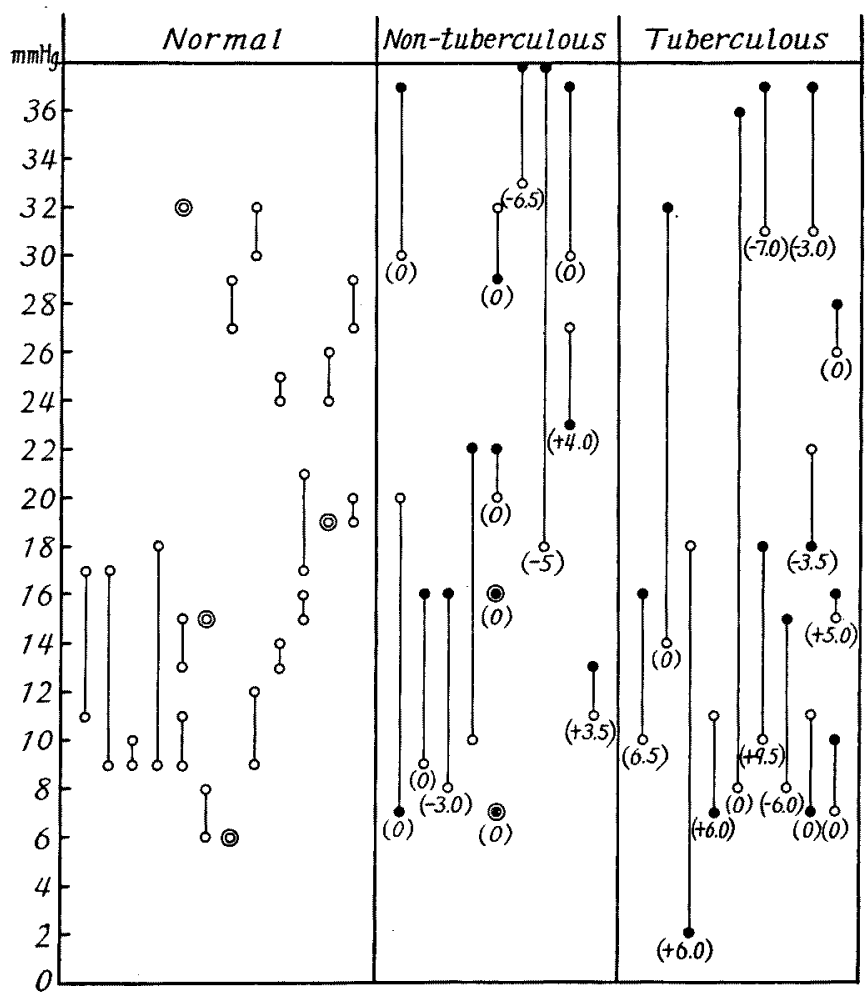

Fig. 1. Relation of the local capillar fragility (C-F) to FTR. 
there was neither quantitative nor qualitative interrelation between the C-F and the FTR $(+)$ or (-), though the G-F usually showed rather large difference between the affected and the healthy side.

2) Resorption test. The disappearance time of intradermally injected salt solution was investigated according to MacGlure and Aldrich's method and the results were correlated with the results of simultaneous FTR tests, but no interrelation was perceptible between them.

3) Diffusion test. The local skin diffusion was tested by injection of trypan-blue to ascertain the possible correlation with the results of simultaneous FTR tests, but no such correlation, either quantitative or qualitative, was found to exist. In 8 tuberculous cases of FTR (dif. +) the results of trypan-blue diffusion appeared equally distributed on both the positive and the negative sides.

\section{Discussion}

From the above experiments, we might conclude that FTR possibly appears in parallel with the local changes in the foci of tuberculous osteoarthritic cases, though with a certain lag in time. From this fact we can derive the value of this test in clinical practice, and we can, in fact, point to many cases where this method was of service in differential as well as focal prognostic diagnosis as we have reported elsewhere. ${ }^{3,6)}$

In the literature, we find scarcely any detailed description concerning the local specific tuberculin reaction of the foci, as given in the above. We can only point to a report of L. Pouyanne ${ }^{10)}$ in 1939 in which he states that the tuberculin reaction works stronger in the skin of the affected side in synovitis cases, and in Japan, to a report by Okaya ${ }^{8)}$ in 1942 about a case where the tuberculin reaction appeared in a stronger degree on the skin of a tuberculous abscess than on the corresponding portion on the healthy side, and to another report by Okazaki ${ }^{9}$ in 1943, in which he remarked that in some cases the results of tuberculin reactions tests were more strongly positive on the affected than on the healthy side in osteoarthritic tuberculosis cases.

The author wishes to propose the following interpreations as to the nature of the FTR. It is accepted that the commonly practiced intracutaneous tuberculin reaction tests involve an antigen-antibody reaction closely related to the so-called Arthus phenomenon. The factors controling the strength of an antigen-antibody reaction are thought to comprise 1) the strength of the antigen, 2) the strength of the antibodies and 3 ) the susceptibility of the local tissue to the reaction, the three items as a whole or a combination of any two items determining the strength of the resultant reaction. The stronger manifestation of FTR in the focal area must also be attributed to the stronger effect of any or all of the 
above three factors. Considering the specificity of tuberculous FTR manifestation, we have to exclude the heightened susceptibility of the local tissue (3 above) and take only the factors 1) and 2) as contributing to the determination of the strength of the FTR by their relative quantity. When both the antigen and antibodies are extremely weak, the resultant reaction between the two will also be weak, when the antigen is incommensurately stronger, the reaction will result in a strong inflammation but the specificity will be lost and closely approximate a normelgic reaction, and when the antibodies are stronger than the antigen beyond all proportion, the reaction will be strangled before any manifestation could take place. Only when an adequate quantity of antigen comes into contact with an adequate quantity of antibodies, a strong and specific reaction will be brought forth. Since in FTR tests, the supplied antigen is constant and consists of $0.1 \mathrm{cc}$. of 2,000th dilution of tuberculin, though the antigen in this case is apt to stagnate in the portion of injection due to the Menkin-Fox effect ${ }^{2,7)}$ and causes a protraction of contact time, the strength of the antigen remains nearly constant throughout, and the factor fortifying the local specific reaction must be sought in a heightened strength of the local anti-tuberculous antibodies. This heightening in the strength or the quantity of antibodies in the tissues near the foci is attributable to the accumulation of specific substances in inflammatory loci, first ascertained with dyes, bacilli etc. by Menkin, ${ }^{7}$ and may better be explained by existence of such an accumulating tendency of antibodies in the circulating blood, as demonstrated by Fox. ${ }^{2}$ Furthurmore, Amano's study') on the subject has set a production of antibodies in tuberculous foci beyond doubt. In particular, the occurrence of medullar intervention and hyperplasia of the adventitial system in osteoarthritic tuberculosis seems to act as a factor in further heightening the density of the local antibodies. The local antibodies thus amplified by accumulation and proliferation will adhere to the cells, chiefly mesenchymal cells, in the vicinity of the foci and thence may sometimes flow into the humor, but it is unquestionable that the density and the strength of the antituberculous antibodies in the vicinity of the foci are distincty higher than those of the humoral generalized antibodies. While these antibodies are subject to strong consumption at the focal reaction field, the elaboration of antigen with locally infesting bacilli and tuberculous tissues promotes also active production of antibodies and the humoral somatic antibodies will tend to accumulate near the focus keeping pace with intensification of the inflammatory change. Thus, it may be assumed that these antibodies are particularly densely adhered to the adjacent tissues immediately connected with the foci. If, in such cases, the proliferation of local antibodies is hampered by some cause and the local inflammation is especially 
intense, the local antibodies will be used up and the local tuberculin reaction will be enfeebled, probably leading to negative FTR results. In such cases, a vehement distruction of tissues may deteriorate reaction activity, and this also occasions negative results in FRT tests. This last kind of negative results indicate the state of negative anergy in FTR, which is observed sometimes not only in tuberculous cases but also in acute suppurative osteomyelitis cases etc.

The cases of such negative FTR are of comparatively rare occurrences, and as in most cases of osteoarthritic tuberculosis a state of relative surplus of antibodies is occasioned in the tissues around the foci, it is easily comprehensible that the part of the skin that is perfused by the blood, the tissue fluid and the lymph that come from or pass near the foci and is histologically in direct connection with the latter has a higher density of antibodies in it than in other parts of the body. Thus, it may be deduced that the FTR (dif. +) cases represent a state where a constant amount of tuberculin is in contact with antibodies in higher concentration in the affected part than in the healthy sites where only generalized somatic antibodies are found. The indication of (dif. -) FTR sometimes observed in the initial stadium of tuberculosis seems to mean the state of weakened tuberculin reaction due to the consumption of antibodies to subnormal levels by the localized tuberculous bacilli and the tuberculous focal antigen, before the proliferation of the antibodies is allowed to take place.

As the reasons why the difference in FTR is distinct only in bone and joint tuberculosis and not so in other tuberculosis cases, such as pulmonary tuberculosis, may be mentioned: 1) Whereas the foci in osteoarthritis tuberculosis are usually situated close to the skin and are histologically connected with the latter, the pulmonary foci are separated from the skin by the pleural cavity etc., 2) the osteoarthritic foci are at a comparatively large distance from the lungs and other major splanchnic organs and are little subjected to the influence of foci in such organs and 3) we can always select an exactly symmetrically corresponding portion on the healthy side as a control.

That FTR is not caused by the difference of susceptibility of the local tissue and that it shows a high specificity has been assured by the absence of the interrelation of FTR with the local capillary fragility, the tissue absorption and diffusion, as has been demonstrated in our auxiliary experiments, and consequently, we believe we can ascribe a significance to FTR as a test for a local reaction specific to tuberculosis.

\section{SUMMARY}

1. The author has ascertained that the tissues, especially the skin, 
in the neighborhood of the foci in osteoarthritic tuberculosis cases are in a state of localized tuberculous allergy specifically different from that in tissues distant from the foci, and the significance of the Focal Tuberculin Reaction (FTR) was discussed.

2. The intracutaneous tuberculin reaction test applied in the skin over a focus of osteoarthritic tuberculosis resulted in a reaction much different in degree from that on the healthy area, and this difference was found to change parallelly with the progress or the stages of the disease. Thus, a test for this difference of FTR is deemed clinically serviceable in differential and prognostic diagnosis. In non-tuberculous orthopedic diseases such a difference of FTR was scarcely observable, except in rheumatic cases.

3. As to the nature of such a difference in FTR, the author's opinion is that it represents a difference in the strength of the reaction owing to a difference in ratio of the antigen to the antibodies in and around the focus. Another factor, a difference in susceptibility of the tissues to the reaction was also considered, but this was found insignificant, both clinically and experimentally.

4. From the above results, we are justified in concluding that FTR is a specific manifestation of the antigen-antibody reaction in the focus of osteo-arthritic tuberculosis.

\section{References}

1) Amano, Ketsuekigaku no Kiso (Jap.), Maruzen Tokyo, 1941.

2) Fox, J. Imm., 1936, 31, 293.

3) Ino, Gekwa (Jap.), 1948, 10, 206.

4) Iino et al., J. Jap. Orthop. Soc. (Jap.), 1949, 23, 113.

5) Idem., ibid., 1950, 24, 49.

6) Kimura, Gekwa (Jap.), 1951, 13, 165.

7) Idem., J. Jap. Orthop. Soc. (Jap.) 1956, 29, 685. (in press)

8) Menkin, J. Exp. Med., 1931, 53, 171.

9) Okaya et al., Jitchi-Ika to Rinsho (Jap.), 1942, 19, 135.

10) Okazaki, Osaka Ijishinshi (Jap.), 1943, 42, 527.

11) Pouyanne, Rev. d'orthop., 1939, 26, 305. 